\title{
CUSTOM MONOTONICITY METHODS
}

\author{
MARTIN SCHECHTER
}

\section{Abstract}

In this paper we show how hypotheses for many problems can be significantly reduced if we employ the monotonicity method. Applications are given.

\section{Introduction}

Consider the problem

$$
-\Delta u=f(x, u), \quad x \in \Omega ; \quad u=0 \text { on } \partial \Omega,
$$

where $\Omega \subset \mathrm{R}^{n}$ is a bounded domain whose boundary is a smooth manifold, and $f(x, t)$ is a continuous function on $\bar{\Omega} \times \mathbf{R}$. In [12], the author and W. Zou assumed the following:

$\left(a_{1}\right)$ There are constants $c_{1}, c_{2} \geq 0$ such that

$$
|f(x, t)| \leq c_{1}+c_{2}|t|^{s},
$$

where $0 \leq s<(n+2) /(n-2)$ if $n>2$.

$\left(a_{2}\right) f(x, t)=o(t)$ as $t \rightarrow 0$, uniformly in $x$.

$\left(a_{3}\right)$ Either

$$
F(x, t) / t^{2} \rightarrow \infty \quad \text { as } \quad t \rightarrow \infty
$$

or

$$
F(x, t) / t^{2} \rightarrow \infty \quad \text { as } \quad t \rightarrow-\infty,
$$

where

$$
F(x, t)=\int_{0}^{t} f(x, s) d s .
$$

We proved

THEOREM 1. Under hypotheses $\left(a_{1}\right),\left(a_{2}\right),\left(a_{3}\right)$ the boundary value problem

$$
-\Delta u=\beta f(x, u), \quad x \in \Omega ; \quad u=0 \text { on } \partial \Omega,
$$

has a nontrivial solution for almost every positive $\beta$.

Received 20 January 2010. 
In this theorem, there was a trade off. The hypothesis $\left(a_{3}\right)$ is significantly weaker than the one usually assumed for superlinear problems. Moreover, the main hypotheses $\left(a_{2}\right),\left(a_{3}\right)$ involve only the primative $F(x, t)$ of $f(x, t)$ rather than $f(x, t)$ itself. This allows much more freedom for the function $f(x, t)$. However, the theorem is proved only for almost every positive $\beta$, not for any particular value of $\beta$. The proof of this theorem was based on the results of our paper [11]. Our method was to use the monotonicity trick introduced by Struwe in [14], [15] for minimization problems. (This trick was also used by others to solve Landesman-Lazer type problems, for bifurcation problems, for Hamiltonian systems and Schrödinger equations.) We applied this method to linking situations.

The purpose of the present paper is to prove the theorem under even weaker assumptions which can allow sublinear problems as well. In particular, we can prove

THEOREM 2. In place of hypothesis $\left(a_{2}\right)$ assume that there is a positive $\tilde{\lambda} \leq \lambda_{0}$ such that

$$
2 F(x, t) \leq \tilde{\lambda} t^{2}, \quad|t| \leq \delta
$$

for some $\delta>0$, and in place of hypothesis $\left(a_{3}\right)$ assume that there are $a \lambda>\tilde{\lambda}$ and an eigenfunction $\varphi$ corresponding to the first eigenvalue $\lambda_{0}$ of $-\Delta u$ such that

$$
\sup _{r>0} \int_{\Omega}\left[v r^{2} \varphi^{2}-2 F(x, r \varphi)\right] d x<\infty
$$

holds for all $v$ satisfying $\tilde{\lambda}<v<\lambda$. Then the boundary value problem

$$
-\Delta u=\beta f(x, u), \quad x \in \Omega ; \quad u=0 \text { on } \partial \Omega,
$$

has a nontrivial solution for almost every $\beta$ in the interval $\left[\lambda_{0} / \lambda, \lambda_{0} / \tilde{\lambda}\right]$.

COROLlary 3. The conclusion of Theorem 2 holds if there is a $\tilde{\lambda} \leq \lambda_{0}$ such that

$$
2 F(x, t) \leq \tilde{\lambda} t^{2}, \quad|t| \leq \delta
$$

for some $\delta>0$ and either

$$
2 F(x, t) \geq \lambda t^{2}-W_{+}(x, t), \quad t>0,
$$

or

$$
2 F(x, t) \geq \lambda t^{2}-W_{-}(x, t), \quad t<0,
$$


holds for some $\lambda>\tilde{\lambda}$, where the $W_{ \pm}(x, t) / t^{2} \leq W(x) \in L^{1}(\Omega)$ satisfy

$$
W_{ \pm}(x, t) / t^{2} \rightarrow 0 \quad \text { a.e. as } t \rightarrow \pm \infty
$$

as the case may be.

Corollary 4. Assume hypotheses $\left(a_{1}\right)$ and

$$
\limsup _{t \rightarrow 0} F(x, t) / t^{2} \leq 0, \quad x \in \Omega .
$$

Assume also that there is an an eigenfunction $\varphi$ corresponding to the first eigenvalue $\lambda_{0}$ of $-\Delta$ u such that (1.4) holds for all positive $v$. Then the boundary value problem

$$
-\Delta u=\beta f(x, u), \quad x \in \Omega ; \quad u=0 \text { on } \partial \Omega,
$$

has a nontrivial solution for almost every positive $\beta$.

Note that Corollaries 3 and 4 allow a wide range of functions $f(x, t)$ both superlinear and sublinear for the conclusion of Theorem 1 to hold for a $\beta$ interval. Also note that it recaptures Theorem 1 in the super-linear case. Our method centers about the construction of a collection $\mathscr{K}_{0}$ of subsets $K$ such that

$$
A \in \mathscr{K}_{0}, \quad B \cap K \neq \phi, \quad K \in \mathscr{K}_{0}
$$

together with

$$
a_{0}:=\sup _{A} G<\infty, \quad b_{0}:=\inf _{B} G>G(0),
$$

holding for a $C^{1}$ functional $G$ implies the existence of a Palais-Smale (PS) sequence, i.e., a sequence $\left\{u_{k}\right\} \subset E$ such that

$$
G\left(u_{k}\right) \rightarrow a, \quad a_{0} \leq a \leq b_{0}, \quad\left\|G^{\prime}\left(u_{k}\right)\right\| \rightarrow 0 .
$$

Our main theorems are presented in Sections 3 and 4. Proofs are given in Section 6 and 7. Applications are given in Section 5.

\section{Flows}

Let $E$ be a Banach space, and let $\Sigma_{0}$ be the set of all continuous maps $\sigma=\sigma(t)$ from $E \times[0,1]$ to $E$ such that

(1) $\sigma(0)$ is the identity map,

(2) for each $t \in[0,1], \sigma(t)$ is a homeomorphism of $E$ onto $E$, 
(3) $\sigma^{\prime}(t)$ is piecewise continuous on $[0,1]$ and satisfies

$$
\left\|\sigma^{\prime}(t) u\right\| \leq \text { const., } \quad u \in E, \quad t \in[0,1],
$$

$$
\sigma(t) 0=0, \quad t \in[0,1] .
$$

The mappings in $\Sigma_{0}$ are called flows. We have customized them for our purposes. The property (2.2) is not usually assumed. We make use of this property in our applications. We note the following.

Remark 5. If $\sigma_{1}, \sigma_{2}$ are in $\Sigma_{0}$, define $\sigma_{3}=\sigma_{1} \circ \sigma_{2}$ by

$$
\sigma_{3}(s)= \begin{cases}\sigma_{1}(2 s), & 0 \leq s \leq \frac{1}{2}, \\ \sigma_{2}(2 s-1) \sigma_{1}(1), & \frac{1}{2}<s \leq 1 .\end{cases}
$$

Then $\sigma_{1} \circ \sigma_{2} \in \Sigma_{0}$.

\section{Sandwich systems}

Let $E$ be a Banach space. We define a nonempty collection $\mathscr{K}_{0}$ of nonempty subsets $K \subset E$ to be a custom sandwich system if $\mathscr{K}_{0}$ has the following property:

$$
\sigma(1) K \in \mathscr{K}_{0}, \quad \sigma \in \Sigma_{0}, \quad K \in \mathscr{K}_{0} .
$$

This property of $\mathscr{K}_{0}$ takes into account the special nature of $\Sigma_{0}$. We have

Theorem 6. Let $\mathscr{K}_{0}$ be a custom sandwich system, and let $G(u)$ be a $C^{1}$ functional on E. Define

$$
a:=\inf _{K \in \mathscr{K}_{0}} \sup _{K} G,
$$

and assume that $a$ is finite and $G(0)<a$. Assume, in addition, that there is a constant $C_{0}$ such that for each $\delta>0$ there is a $K \in \mathscr{K}_{0}$ satisfying

$$
\sup _{K} G \leq a+\delta,
$$

such that the inequality

$$
G(u) \geq a-\delta, \quad u \in K,
$$

implies $\|u\| \leq C_{0}$. Then there is a bounded PS sequence $\left\{u_{k}\right\} \subset E$ such that

$$
G\left(u_{k}\right) \rightarrow a, \quad\left\|G^{\prime}\left(u_{k}\right)\right\| \rightarrow 0 .
$$


The advantage of this theorem is the fact that for most applications there is need to add appropriate hypotheses to obtain a convergent subsequence. This is usually achieved by hypotheses that cause the PS sequence to be bounded. Theorem 6 obviates this requirement.

Theorem 7. Let $\mathscr{K}_{0}$ be a custom sandwich system, and let $G(u)$ be a $C^{1}$ functional on E. Assume that there are subsets $A, B$ of $E$ such that

$$
a_{0}:=\sup _{A} G<\infty, \quad b_{0}:=\inf _{B} G>G(0),
$$

$A \in \mathscr{K}_{0}$ and

$$
B \cap K \neq \phi, \quad K \in \mathscr{K}_{0} .
$$

Then a given by (3.1) satisfies $b_{0} \leq a \leq a_{0}$. Assume, in addition, that there is a constant $C_{0}$ such that for each $\delta>0$ there is a $K \in \mathscr{K}_{0}$ satisfying (3.2) such that the inequality (3.3) implies $\|u\| \leq C_{0}$. Then there is a bounded sequence $\left\{u_{k}\right\} \subset$ E satisfying (3.4).

Definition 8. We shall say that sets $A, B$ in $E$ form a custom sandwich pair if $A$ is a member of a custom sandwich system $\mathscr{K}_{0}$ and $B$ satisfies (3.6).

We have

TheOREM 9. Let $A$ be a continuous curve in E connecting $O$ and $\infty$, and let $B$ the boundary of a bounded open set in $E$ containing 0 . Then $A, B$ form a custom sandwich pair.

\section{The parameter problem}

Let $E$ be a reflexive Banach space with norm $\|\cdot\|$. Suppose that $G \in \mathscr{C}^{1}(E, \mathrm{R})$ is of the form: $G(u):=I(u)-J(u), u \in E$, where $I, J \in \mathscr{C}^{1}(E, \mathrm{R})$ map bounded sets to bounded sets. Define

$$
G_{\lambda}(u)=\lambda I(u)-J(u), \quad \lambda \in \Lambda,
$$

where $\Lambda$ is an open interval contained in $(0,+\infty)$. Assume one of the following alternatives holds.

$\left(\mathrm{H}_{1}\right) \quad I(u) \geq 0$ for all $u \in E$ and $I(u)+|J(u)| \rightarrow \infty$ as $\|u\| \rightarrow \infty$, or

$\left(\mathrm{H}_{2}\right) \quad I(u) \leq 0$ for all $u \in E$ and $|I(u)|+|J(u)| \rightarrow \infty$ as $\|u\| \rightarrow \infty$.

Furthermore, we suppose that $\mathscr{K}_{0}$ is a custom sandwich system satisfying $\left(\mathrm{H}_{3}\right) a(\lambda):=\inf _{K \in \mathscr{Y}_{0}} \sup _{K} G_{\lambda}<\infty$ for any $\lambda \in \Lambda$. 
Theorem 10. Assume that $\left(\mathrm{H}_{1}\right)\left(\right.$ or $\left.\left(\mathrm{H}_{2}\right)\right)$ and $\left(\mathrm{H}_{3}\right)$ hold. Then we have

(1) For almost all $\lambda \in \Lambda$ there exists a constant $k_{0}(\lambda):=k_{0}$ (depending only on $\lambda$ ) such that for each $\delta>0$ there exists a $K \in \mathscr{K}_{0}$ such that $\sup _{K} G_{\lambda} \leq a(\lambda)+\delta$ and

$$
\|u\| \leq k_{0} \quad \text { whenever } \quad u \in K \text { and } G_{\lambda}(u) \geq a(\lambda)-\delta .
$$

(2) For almost all $\lambda \in \Lambda$ there exists a bounded sequence $u_{k}(\lambda) \in E$ such that

$$
\left\|G_{\lambda}^{\prime}\left(u_{k}\right)\right\| \rightarrow 0, \quad G_{\lambda}\left(u_{k}\right) \rightarrow a(\lambda):=\inf _{K \in \mathscr{K}_{0}} \sup _{K} G_{\lambda}, \quad k \rightarrow \infty .
$$

COROLlaRY 11. The conclusions of Theorem 10 hold if we replace hypothesis $\left(\mathrm{H}_{3}\right)$ with

$\left(\mathrm{H}_{3}^{\prime}\right)$ There is a custom sandwich pair A, B such that

$$
a_{0}:=\sup _{A} G_{\mu}<\infty, \quad G_{\mu}(0)<b_{0}:=\inf _{B} G_{\mu}
$$

for each $\mu \in \Lambda$.

\section{Some applications}

Many elliptic semi-linear problems can be described in the following way. Let $\Omega$ be a domain in $\mathrm{R}^{n}$, and let $\mathscr{A}$ be a self-adjoint operator on $L^{2}(\Omega)$. We assume that $\mathscr{A} \geq \lambda_{0}>0$ and that

$$
C_{0}^{\infty}(\Omega) \subset D:=D\left(\mathscr{A}^{1 / 2}\right) \subset H^{m, 2}(\Omega)
$$

for some $m>0$, where $C_{0}^{\infty}(\Omega)$ denotes the set of test functions in $\Omega$ (i.e., infinitely differentiable functions with compact supports in $\Omega$ ), and $H^{m, 2}(\Omega)$ denotes the Sobolev space. If $m$ is an integer, the norm in $H^{m, 2}(\Omega)$ is given by

$$
\|u\|_{m, 2}:=\left(\sum_{|\mu| \leq m}\left\|D^{\mu} u\right\|^{2}\right)^{1 / 2}
$$

Here $D^{\mu}$ represents the generic derivative of order $|\mu|$ and the norm on the right hand side of (5.2) is that of $L^{2}(\Omega)$. We shall assume that $m$ is an integer. As an example of such an operator, we can take $\mathscr{A}$ to be an elliptic partial differential operator of the form

$$
\mathscr{A}=\sum_{|\mu| \leq 2 m} a_{\mu}(x) D^{\mu},
$$


with sufficiently smooth coefficients. If

$$
(\mathscr{A} u, u) \geq \lambda_{0}\|u\|^{2}, \quad u \in H^{m, 2}(\Omega),
$$

with $\lambda_{0}>0$, then $\mathscr{A}$ has a selfadjoint extension satisfying the hypotheses given above.

Let $q$ be any number satisfying

$$
\begin{aligned}
& 2<q<2 n /(n-2 m), \quad 2 m<n, \\
& 2<q<\infty, \quad n \leq 2 m,
\end{aligned}
$$

and let $f(x, t)$ be a Carathéodory function on $\Omega \times \mathrm{R}$. This means that $f(x, t)$ is continuous in $t$ for a.e. $x \in \Omega$ and measurable in $x$ for every $t \in \mathrm{R}$. Throughout this section we make the following assumptions:

(A) The function $f(x, t)$ satisfies

$$
|f(x, t)| \leq V_{0}(x)^{q}|t|^{q-1}+V_{0}(x)^{q} W_{0}(x)
$$

and

$$
f(x, t) / V_{0}(x)^{q}=o\left(|t|^{q-1}\right) \quad \text { as } \quad|t| \rightarrow \infty,
$$

where $V_{0}(x)>0$ is a function such that

$$
\left\|V_{0} u\right\|_{q} \leq C\|u\|_{D}, \quad u \in D
$$

and $W_{0}$ is a function in $L^{\infty}(\Omega)$. Here

$$
\|u\|_{q}:=\left(\int_{\Omega}|u(x)|^{q} d x\right)^{1 / q},
$$

and

$$
\|u\|_{D}:=\left\|\mathscr{A}^{1 / 2} u\right\| .
$$

If $\Omega$ and $V_{0}(x)$ are bounded, then (5.6) will hold automatically by the Sobolev inequality. However, there are functions $V_{0}(x)$ which are unbounded and such that (5.6) holds even on unbounded regions $\Omega$. With the norm (5.8), $D$ becomes a Hilbert space. Define

$$
F(x, t):=\int_{0}^{t} f(x, s) d s
$$


and

$$
G(u):=\|u\|_{D}^{2}-2 \int_{\Omega} F(x, u) d x .
$$

It follows that $G$ is a continuously differentiable functional on the whole of $D$ (cf., e.g., [6]).

For $\mu>0$, we let

$$
G_{\mu}(u):=\mu\|u\|_{D}^{2}-2 \int_{\Omega} F(x, u) d x .
$$

We wish to obtain a solution of

$$
\mu \mathscr{A} u=f(x, u), \quad u \in D
$$

By a solution of (5.12) we shall mean a function $u \in D$ such that

$$
\mu(u, v)_{D}=(f(\cdot, u), v), \quad v \in D .
$$

If $f(x, u)$ is in $L^{2}(\Omega)$, then a solution of (5.13) is in $D(\mathscr{A})$ and solves (5.12) in the classical sense. Otherwise we call it a weak (or semi-strong) solution.

We assume that $\lambda_{0}$ is a simple isolated eigenvalue of $\mathscr{A}$ having a bounded eigenfunction $\varphi(x)$. In addition, we assume that there is a positive number $\tilde{\lambda} \leq \lambda_{0}$ such that

$$
2 F(x, t) \leq \tilde{\lambda} t^{2}, \quad|t|<\delta
$$

for some positive constant $\delta$. Moreover, we assume that

$$
\sup _{r>0} \int_{\Omega}\left[v r^{2} \varphi^{2}-2 F(x, r \varphi)\right] d x<\infty
$$

for each $v$ satisfying $\tilde{\lambda}<v<\lambda$ for some $\lambda>\tilde{\lambda}$. We have

THEOREM 12. Under the above hypotheses, the equation

$$
\mathscr{A} u=\beta f(x, u), \quad u \in D
$$

has at least one nontrivial solution for almost all $\beta \in\left[\lambda_{0} / \lambda, \lambda_{0} / \tilde{\lambda}\right]$.

Proof. We apply Corollary 11 . We let $N$ be the eigenspace $E\left(\lambda_{0}\right)$, and we take $M=N^{\perp}$. We note that (5.14) implies

$$
G_{\mu}(u) \geq(\mu-\eta) \rho^{2}, \quad\|u\|_{D}=\rho
$$


for $\rho>0$ sufficiently small, where $\eta=\tilde{\lambda} / \lambda_{0}$. To see this, let $u=w+y$, where $w \in M$ and $y \in N$. Note that there is a $\rho>0$ such that

$$
\|y\|_{D} \leq \rho \Rightarrow|y(x)| \leq \delta / 2, \quad y \in E\left(\lambda_{0}\right) .
$$

Now suppose $u$ satisfies

$$
\|u\|_{D} \leq \rho \quad \text { and } \quad|u(x)| \geq \delta
$$

for some $x \in \Omega$. Then for those $x \in \Omega$ satisfying (5.18) we have

$$
\delta \leq|u(x)| \leq|w(x)|+|y(x)| \leq|w(x)|+(\delta / 2) .
$$

Hence

$$
|y(x)| \leq \delta / 2 \leq|w(x)|
$$

and consequently,

$$
|u(x)| \leq 2|w(x)|
$$

for all such $x$. Now we have by hypothesis (A) and (5.14)

$$
\begin{aligned}
G_{\mu}(u) & \geq \mu\|u\|_{D}^{2}-\tilde{\lambda} \int_{|u|<\delta} u^{2} d x-C \int_{|u|>\delta}\left(|V u|^{q}+V^{q}|u|\right) d x \\
& \geq \mu\|u\|_{D}^{2}-\tilde{\lambda}\|u\|^{2}-C^{\prime} \int_{|u|>\delta}|V u|^{q} d x \\
& \geq(\mu-\eta)\|y\|_{D}^{2}+\mu\|w\|_{D}^{2}-\tilde{\lambda}\|w\|^{2}-C^{\prime \prime} \int_{2|w|>\delta}|V w|^{q} d x
\end{aligned}
$$

in view of the fact that $\|y\|_{D}^{2}=\lambda_{0}\|y\|^{2}$ and (5.19) holds. Thus, by (5.6),

$$
G_{\mu}(u) \geq(\mu-\eta)\|y\|_{D}^{2}+\left(\mu-\frac{\tilde{\lambda}}{\lambda_{1}}-C^{\prime \prime \prime}\|w\|_{D}^{q-2}\right)\|w\|_{D}^{2}, \quad\|u\|_{D} \leq \rho,
$$

where $\lambda_{1}$ is the next point in the spectrum of $\mathscr{A}$. We take $\rho>0$ to satisfy

$$
\eta-\frac{\tilde{\lambda}}{\lambda_{1}}>C^{\prime \prime \prime} \rho^{q-2}
$$

Consequently,

$$
G_{\mu}(u) \geq(\mu-\eta) \rho^{2}+\left(\mu-\frac{\tilde{\lambda}}{\lambda_{1}}-C^{\prime \prime \prime} \rho^{q-2}-\mu+\eta\right)\|w\|_{D}^{2} \geq(\mu-\eta) \rho^{2},
$$

$\|u\|_{D}=\rho$. Thus, (5.17) holds. 
We let $A=\{r \varphi: r \geq 0\}$ and $B=\partial \mathbf{B}_{\rho}$. By Theorem 9, they form a custom sandwich pair. Note that

$$
G_{\mu}(r \varphi)=\int_{\Omega}\left[\mu \lambda_{0} r^{2} \varphi^{2}-2 F(x, r \varphi)\right] d x .
$$

By (5.15) and (5.17), we see that (4.2) holds for each $G_{\mu}$, for $\tilde{\lambda} / \lambda_{0}<\mu<\lambda / \lambda_{0}$ with $b_{0}>0$. Apply Corollary 11 , and take $\beta=1 / \mu$.

Proof of Theorem 2. Since the Dirichlet problem (1.2) is a special case of problem (5.16), Theorem 12 implies Theorem 2.

Proof of Corollary 3. We know that $\varphi$ does not change sign in $\Omega$. We take it to be positive and satisfy $\|\varphi\|_{D}=1$. Since

$$
G_{\mu}( \pm r \varphi)=\int_{\Omega}\left[\mu \lambda_{0} r^{2} \varphi^{2}-2 F(x, \pm r \varphi)\right] d x,
$$

we have by (1.7) or (1.8)

$$
G_{\mu}( \pm r \varphi) / r^{2} \leq \mu \lambda_{0}-\lambda+\int_{\Omega}\left[W_{ \pm}(x, \pm r \varphi) / r^{2} \varphi^{2}\right] \varphi^{2} d x \rightarrow \mu \lambda_{0}-\lambda<0
$$

as $r \rightarrow \infty$, as the case may be. This shows that (4.2) holds. Apply Corollary 11 .

\section{Finding the sequences}

We proceed to the proof of Theorem 6. Let $M=C_{0}+1$. Then

$$
\|\sigma(1) v\| \leq M
$$

whenever $\sigma \in \Sigma_{0}$ satisfies $\left\|\sigma^{\prime}(t)\right\| \leq 1$ and $v \in E$ satisfies $\|v\| \leq C_{0}$. If the theorem were false, then there would be a $\delta>0$ such that

$$
\left\|G^{\prime}(u)\right\| \geq 3 \delta
$$

when

$$
u \in\{u \in E:\|u\| \leq M+1,|G(u)-a| \leq 3 \delta\} .
$$

Take $\delta<1 / 3$ so small that $G(0)<b_{0}-2 \delta$. Since $G \in C^{1}(E, \mathrm{R})$, for each $\theta<1$ there is a locally Lipschitz continuous mapping $Y(u)$ of $\hat{E}=$ $\left\{u \in E: G^{\prime}(u) \neq 0\right\}$ into $E$ such that

$$
\|Y(u)\| \leq 1, \quad \theta\left\|G^{\prime}(u)\right\| \leq\left(G^{\prime}(u), Y(u)\right), \quad u \in \hat{E}
$$


(cf., e.g., [6]). Take $\theta>2 / 3$. Let

$$
\begin{aligned}
Q_{0} & =\{u \in E:\|u\| \leq M+1,|G(u)-a| \leq 2 \delta\}, \\
Q_{1} & =\{u \in E:\|u\| \leq M,|G(u)-a| \leq \delta\}, \\
Q_{2} & =E \backslash Q_{0}, \\
\eta(u) & =d\left(u, Q_{2}\right) /\left[d\left(u, Q_{1}\right)+d\left(u, Q_{2}\right)\right] .
\end{aligned}
$$

It is easily checked that $\eta(u)$ is locally Lipschitz continuous on $E$ and satisfies

$$
\begin{cases}\eta(u)=1, & u \in Q_{1}, \\ \eta(u)=0, & u \in \bar{Q}_{2}, \\ \eta(u) \in(0,1), & \text { otherwise. }\end{cases}
$$

Let

$$
W(u)=-\eta(u) Y(u)
$$

Then

$$
\|W(u)\| \leq 1, \quad u \in E .
$$

By Theorem 4.5 of [7], for each $v \in E$ there is a unique solution $\sigma(t) v$ of

$$
\sigma^{\prime}(t)=W(\sigma(t)), \quad t \in \mathrm{R}^{+}, \sigma(0)=v .
$$

We have

$$
\begin{aligned}
d G(\sigma(t) v) / d t & =-\eta(\sigma(t) v)\left(G^{\prime}(\sigma(t) v), Y(\sigma(t) v)\right) \\
& \leq-\theta \eta(\sigma)\left\|G^{\prime}(\sigma)\right\| \\
& \leq-3 \theta \delta \eta(\sigma) .
\end{aligned}
$$

Let $K \in \mathscr{K}_{0}$ satisfy the hypotheses of the theorem. Let $v$ be any element of $K \cap Q_{1}$. Then $\|v\| \leq C_{0}$. If there is a $t_{1} \leq 1$ such that $\sigma\left(t_{1}\right) v \notin Q_{1}$, then

$$
G(\sigma(1) v)<a-\delta
$$

since $\|\sigma(1) v\| \leq M$,

$$
G(\sigma(1) v) \leq G\left(\sigma\left(t_{1}\right) v\right)
$$

and the right hand side cannot be greater than $a+\delta$ by (6.6). On the other hand, if $\sigma(t) v \in Q_{1}$ for all $t \in[0,1]$, then we have by (6.6)

$$
G(\sigma(1) v) \leq a+\delta-3 \delta \theta<a-\delta .
$$

If $v \in K \backslash Q_{1}$, then we must have

$$
G(\sigma(1) v) \leq G(v)<a-\delta,
$$


since $G(v) \geq a-\delta$ would put $v$ into $Q_{1}$. Hence

$$
G(\sigma(1) v)<a-\delta, \quad v \in K .
$$

Next, we note that $\sigma \in \Sigma_{0}$. To see this, note that $\eta(u)=0$ when $G(u)<a-2 \delta$. Since $G(0)<a-2 \delta$ and $G(\sigma(t) 0)$ decreases as $t$ increases, we see that $\sigma^{\prime}(t) 0=0$ for $t \in[0,1]$. By hypothesis, $\tilde{K}=\sigma(1) K \in \mathscr{K}_{0}$. This means that

$$
G(w)<a-\delta, \quad w \in \tilde{K} .
$$

But this contradicts the definition (3.1) of $a$. Hence (6.1) cannot hold for $u$ satisfying (6.2). This proves the theorem.

Proof of Theorem 7. Since $A \in \mathscr{K}_{0}$, clearly $a \leq a_{0}$, Moreover, for any $K \in \mathscr{K}_{0}$, we have

$$
b_{0}=\inf _{B} G \leq \inf _{B \cap K} G \leq \sup _{B \cap K} G \leq \sup _{K} G .
$$

Hence, $b_{0} \leq a$. Apply Theorem 6 .

Proof of Theorem 9. Take

$$
\mathscr{K}_{0}=\left\{\sigma(1) A: \sigma \in \Sigma_{0}\right\} .
$$

If $\sigma \in \Sigma_{0}$ and $v \in A \cap \partial B_{R}$, then

$$
\|\sigma(1) v-v\|=\left\|\int_{0}^{1} \sigma^{\prime}(t) v d t\right\| \leq 1 .
$$

Consequently,

$$
\|\sigma(1) v\| \geq\|v\|-\|\sigma(1) v-v\| \geq R-1 \rightarrow \infty \quad \text { as } \quad R \rightarrow \infty .
$$

Since $\sigma(1) 0=0$, we see that $\sigma(1) A \cap B \neq \phi$.

\section{The monotonicity trick}

We now give the proof of Theorem 10.

Proof. We prove conclusion (1) assuming the first alternative hypothesis $\left(\mathrm{H}_{1}\right)$.

By $\left(\mathrm{H}_{1}\right)$, the map $\lambda \mapsto a(\lambda)$ is nondecreasing. Hence, $a^{\prime}(\lambda):=d a(\lambda) / d \lambda$ exists for almost every $\lambda \in \Lambda$. From this point on, we consider those $\lambda$ where $a^{\prime}(\lambda)$ exists. For fixed $\lambda \in \Lambda$, let $\lambda_{n} \in(\lambda, 2 \lambda) \cap \Lambda, \lambda_{n} \rightarrow \lambda$ as $n \rightarrow \infty$. Then there exists $\bar{n}(\lambda)$ such that

$$
a^{\prime}(\lambda)-1 \leq \frac{a\left(\lambda_{n}\right)-a(\lambda)}{\lambda_{n}-\lambda} \leq a^{\prime}(\lambda)+1 \quad \text { for } \quad n \geq \bar{n}(\lambda) .
$$


Next, we note that there exist $K_{n} \in \mathscr{K}_{0}, k_{0}:=k_{0}(\lambda)>0$ such that

$$
\|u\| \leq k_{0} \quad \text { whenever } \quad G_{\lambda}(u) \geq a(\lambda)-\left(\lambda_{n}-\lambda\right) .
$$

In fact, by the definition of $a\left(\lambda_{n}\right)$, there exists $K_{n}$ such that

$$
\sup _{K_{n}} G_{\lambda}(u) \leq \sup _{K_{n}} G_{\lambda_{n}}(u) \leq a\left(\lambda_{n}\right)+\left(\lambda_{n}-\lambda\right) .
$$

If $G_{\lambda}(u) \geq a(\lambda)-\left(\lambda_{n}-\lambda\right)$ for some $u \in K_{n}$, then, by (7.1) and (7.3), we have that

$$
\begin{aligned}
I(u) & =\frac{G_{\lambda_{n}}(u)-G_{\lambda}(u)}{\lambda_{n}-\lambda} \\
& \leq \frac{a\left(\lambda_{n}\right)+\left(\lambda_{n}-\lambda\right)-a(\lambda)+\left(\lambda_{n}-\lambda\right)}{\lambda_{n}-\lambda} \\
& \leq a^{\prime}(\lambda)+3,
\end{aligned}
$$

and it follows that

$$
\begin{aligned}
J(u) & =\lambda_{n} I(u)-G_{\lambda_{n}}(u) \\
& \leq \lambda_{n}\left(a^{\prime}(\lambda)+3\right)-G_{\lambda}(u) \\
& \leq \lambda_{n}\left(a^{\prime}(\lambda)+3\right)-a(\lambda)+\left(\lambda_{n}-\lambda\right) \\
& \leq 2 \lambda\left(a^{\prime}(\lambda)+3\right)-a(\lambda)+\lambda .
\end{aligned}
$$

On the other hand, by $\left(\mathrm{H}_{1}\right),(7.1)$, and (7.3),

$$
\begin{aligned}
J(u) & =\lambda_{n} I(u)-G_{\lambda_{n}}(u) \\
& \geq-G_{\lambda_{n}}(u) \\
& \geq-\left(a\left(\lambda_{n}\right)+\left(\lambda_{n}-\lambda\right)\right) \\
& \geq-\left(a(\lambda)+\left(\lambda_{n}-\lambda\right)\left(a^{\prime}(\lambda)+2\right)\right) \\
& \geq-a(\lambda)-\lambda\left|a^{\prime}(\lambda)+2\right| .
\end{aligned}
$$

Combining (7.4)-(7.6) and $\left(\mathrm{H}_{1}\right)$, we see that there exists $k_{0}(\lambda):=k_{0}$ (depending only on $\lambda$ ) such that (7.2) holds.

By the choice of $K_{n}$ and (7.1), we see that

$$
\begin{aligned}
G_{\lambda}(u) & \leq G_{\lambda_{n}}(u) \\
& \leq \sup _{K_{n}} G_{\lambda_{n}}(u) \\
& \leq a\left(\lambda_{n}\right)+\left(\lambda_{n}-\lambda\right) \\
& \leq\left(a^{\prime}(\lambda)+1\right)\left(\lambda_{n}-\lambda\right)+a(\lambda)+\left(\lambda_{n}-\lambda\right) \\
& \leq a(\lambda)+\left(a^{\prime}(\lambda)+2\right)\left(\lambda_{n}-\lambda\right)
\end{aligned}
$$


for all $u \in K_{n}$. We take $n$ sufficiently large to ensure that $\left|a^{\prime}(\lambda)+2\right|\left(\lambda_{n}-\lambda\right)<\delta$. This proves conclusion (1). Conclusion (2) now follows from Theorem 6 . The proof under hypothesis $\left(\mathrm{H}_{2}\right)$ is similar, and is omitted.

\section{REFERENCES}

1. Furtado, M. F., Maia, L. A., and Silva, E. A. B., On a double resonant problem in $\mathrm{R}^{N}$, Differential Integral Equations 15 (2002), 1335-1344.

2. Furtado, M. F., and Silva, E. A. B., Double resonant problems which are locally non-quadratic at infinity, pp. 155-171 in: Proceedings of the USA-Chile Workshop on Nonlinear Analysis (Proc. Viña del Mar-Valparaiso 2000), Electron. J. Differ. Equ. Conf. 6, Southwest Texas State Univ., San Marcos, TX 2001.

3. Jeanjean, L., On the existence of bounded Palais-Smale sequences and application to a Landesman-Lazer type problem set on $\mathbf{R}^{N}$, Proc. Roy. Soc. Edinburgh (A) 129 (1999), 787-809.

4. Jeanjean, L., Local conditions insuring bifurcation from the continuous spectrum, Math. Z. 232 (1999), 651-664.

5. Schechter, M., A generalization of the saddle point method with applications, Ann. Polon. Math. 57 (1992), 269-281.

6. Schechter, M., Linking Methods in Critical Point Theory, Birkhäuser, Boston 1999.

7. Schechter, M., Minimax Systems and Critical Point Theory, Birkhäuser, Boston 2009.

8. Schechter, M., New saddle point theorems, pp. 213-219 in: Generalized Functions and their Applications (Proc. Varanasi 1991), Plenum, New York 1993.

9. Schechter, M., Sandwich pairs in critical point theory, Trans. Amer. Math. Soc. 360 (2008), 2811-2823.

10. Schechter, M., Strong sandwich pairs, Indiana Univ. Math. J. 57 (2008), 1105-1131.

11. Schechter, M., and Zou, W., Double linking theorem and multiple solutions, J. Funct. Anal. 205 (2003), 37-61.

12. Schechter, M., and Zou, W., Superlinear problems, Pacific J. Math. 214 (2004), 145-160.

13. Silva, E. A. de B. e, Linking theorems and applications to semilinear elliptic problems at resonance, Nonlinear Anal. 16 (1991) 455-477.

14. Struwe, M., The existence of surfaces of constant mean curvature with free boundaries, Acta Math. 160 (1988), 19-64.

15. Struwe, M., Variational Methods - Applications to Nonlinear Partial Differential Equations and Hamiltonian Systems, 2nd ed., Ergebnisse Math. Grenzgebiete (3) 34, Springer, Berlin 1996.

16. Willem, M., and Zou, W., On a semilinear Dirichlet problem and a nonlinear Schrödinger equation with periodic potential, preprint.

DEPARTMENT OF MATHEMATICS

UNIVERSITY OF CALIFORNIA

IRVINE, CA 92697-3875

U.S.A

E-mail: mschecht@math.uci.edu 